\section{Neurofibromatosis type I and multiple myeloma coexistence: A possible link?}

\author{
Fabrizio Accardi ${ }^{1,2}$ \\ Valentina Marchica, ${ }^{1}$ Cristina Mancini, ${ }^{3}$ \\ Elena Maredi, ${ }^{4}$ Costantina Racano, ${ }^{4}$ \\ Laura Notarfranchi, ${ }^{1,2}$ \\ Davide Martorana, ${ }^{5}$ Paola Storti, ${ }^{1}$ \\ Eugenia Martella, ${ }^{3}$ \\ Benedetta Dalla Palma, ${ }^{1,2}$ \\ Luisa Craviotto, ${ }^{1,2}$ Massimo De Filippo, ${ }^{6}$ \\ Antonio Percesepe, ${ }^{5}$ Franco Aversa, ${ }^{1,2}$ \\ Nicola Giuliani $\mathbf{1}^{1,2}$ \\ ${ }^{1}$ Department of Medicine and Surgery, \\ University of Parma; ${ }^{2} \mathrm{Hematology}$ Unit, \\ Parma University Hospital; ${ }^{3}$ Pathology \\ Unit, Parma University Hospital; \\ ${ }^{4}$ Pediatric Orthopedics, Rizzoli \\ Orthopedic Institute, Bologna; ${ }^{5}$ Medical \\ Genetics, Parma University Hospital; \\ ${ }^{6}$ Radiology Unit, University of Parma, \\ Italy
}

\begin{abstract}
The association between Neurofibromatosis type I (NF1) and multiple myeloma (MM), a plasma cell, dyscrasia is very rare. Here we put to the attention of the scientific community two new cases. The first one is a patient with active MM whereas the second with smoldering MM. Both patients present typical features of NF1 but skeletal alterations were present only in the second case including dysplasia, marked scoliosis and osteoporosis. MM osteolytic lesions were absent in both patients. In addition to the clinical diagnosis of NF1, a molecular testing for NF1 gene mutations has been performed finding that patient one was heterozygous for the c. $6855 \mathrm{C}>\mathrm{A}$ (Tyr2285Ter) mutation, while patient two was heterozygous for the c.7838dupC (Lys2614GlufsTer20) mutation. The two mutations were diagnosed both in genomic DNA from peripheral blood and from MM cells. The potential link between NF1 mutation and the increased risk of $\mathrm{MM}$ is discussed in the report.
\end{abstract}

\section{Introduction}

Neurofibromatosis type I (NF1) is an inherited autosomal dominant condition which affects approximately one in 2500 to one in 3000 people. ${ }^{1}$ This genetic disorder originates from germline mutation in NFI gene, located on chromosome 17q11.2, which encodes for neurofibromin, a GTPase activating protein (GAP), that acts as a negative regulator of the RAS transduction signal pathway through the increase of Ras GTP-ase activity, leading to Ras inactivation in the GDP-bound conformation. Lossof-function mutations of $N F 1$ gene result in higher activation of the Ras signaling and downstream mitogen-activated protein kinase (MAPK) pathway, enhancing cell growth and proliferation. ${ }^{2}$ NF1 belongs to the so-called RASopathies, which include different genetic disorders, conferring a predisposition to cancer development. ${ }^{2}$

Bone dysplasia in NF1 patients is the most common alteration and includes that of the tibia present in about 3-4\% of patients. ${ }^{3}$ Spinal deformity, reported in approximately $49 \%$ of patients with NF1, has been traditionally classified as dystrophic or non-dystrophic based on radiographic findings. In adults and children with NF1, decreased bone mineral density (BMD) and increased fracture rates are also typically observed. Stevenson et al. ${ }^{4}$ demonstrated that osteopenia characterizes NF1 patients suggesting that there is a generalized bone abnormality in NF1, and that NF1 haploinsufficiency potentially impacts bone cellular functions. ${ }^{3,4}$ Indeed higher osteoclast formation and activity have been reported in NF1 patients. ${ }^{5,6}$

Patients with NF1 are susceptible to develop benign and malignant tumors of the nervous system. Furthermore, other sporadic cancer types such as breast cancer, gastrointestinal stromal tumor (GIST), pheochromocytoma, acute myeloid leukemia and juvenile myelomonocytic leukemia (JMML) are associated with NF1. ${ }^{7,8}$ However, the association with multiple myeloma (MM) is very rare and to the best of our knowledge only three single cases have been reported in the English literature. ${ }^{9-11}$

\section{Case Report \#1}

A 59-year-old man was evaluated in July 2013 for progressive lumbar pain and lower limb weakness for about two months. His medical history included cholecystectomy and resection of 2 formations compatible with neurofibroma in the right anterior mediastinum and in the right ankle when 45 years old. Paternal clinical history was positive for cutaneous neurofibromas. Examination disclosed several dermatological signs such as small movable nodules located on the face, trunk and arms, a palpa-
Correspondence: Nicola Giuliani, Department of Medicine and Surgery, University of Parma, Via Gramsci 14, 43126, Parma, Italy. Tel.: +39.052.1033299 - Fax: +39.052.1033264. E-mail: nicola.giuliani@unipr.it

Key words: Multiple Myeloma, Neurofibromatosis, NF1.

Contributions: FA and NG wrote the manuscript; FA, NG, LN, LC and FA analyzed clinical data of the patients; $\mathrm{CM}$ and EM performed immunohistochemistry; VM, PS, DV and $\mathrm{AP}$ performed genetic study; $\mathrm{MD}, \mathrm{CR}$, and EM analyzed skeletal imaging.

Conflict of interest: the authors declare no potential conflict of interest.

Funding: this work was supported in part by a grant from the Associazione Italiana per la Ricerca sul Cancro (AIRC) IG2014 n.15531 (NG)

Received for publication: 24 October 2017. Accepted for publication: 28 February 2018.

This work is licensed under a Creative Commons Attribution-NonCommercial 4.0 International License (CC BY-NC 4.0).

(C) Copyright F. Accardi et al., 2018

Licensee PAGEPress, Italy

Hematology Reports 2018; 10:7457

doi:10.4081/hr.2018.7457

ble subcutaneous lesion of $3 \mathrm{~cm}$ on the right of the neck (Figure 1A). Ophthalmologic examination detected Lisch nodules in the iris. A diagnosis of NF1 according to NIH consensus criteria was formulated. ${ }^{1}$ Magnetic resonance imaging (MRI) of the spine identified a dumbbell shaped lesion on L3 level consistent with paraspinal neurofibroma, vertebral collapse on L2 and a diffuse pattern of the vertebral bodies, congruous with MM involvement (Figure 1B). Routine laboratory tests showed $10.3 \mathrm{gr} / \mathrm{dL}$ hemoglobin, increased ESR, normal creatinine, electrolytes and liver function, hyperproteinemia and a monoclonal component IgG-kappa. Bone marrow (BM) aspirate contained $50 \%$ of plasma cells (PCs) infiltration confirming the diagnosis of multiple myeloma. Skeletal survey was negative for lytic lesions.

The patient underwent vertebroplasty and was treated with a bortezomib-based induction regimen VTD (bortezomibthalidomide-dexamethasone) for 2 cycles with a minor response and $70 \%$ of PCs were detected in a new BM biopsy. Therein, he was treated with two further VTD cycles, with the addition of oral cyclophosphamide, obtaining a partial response (PR) according 
to IMWG criteria and later proceeded with stem collection and tandem autologous bone marrow transplantation followed by a VTD consolidation treatment reaching a very good partial response (VGPR). The patient also received monthly zoledronic acid $4 \mathrm{mg}$ intravenous for two years. No bone lesions or fracture occurred during the follow up and currently the patient remains in VGPR. Further analysis was performed for the biological characterization of the patient. Fluorescence in situ hybridization (FISH) analysis on purified CD138 ${ }^{+} \mathrm{PCs}$ identified the presence of $\operatorname{del}(13 q)$ and trisomy of chromosome 9 and 15 but did not show del(17p) and chromosome 14 rearrangements. Markers of bone remodeling including Activin A, Dickkopf-1 (DKK1), C-C motif Ligand (CCL) 3 and CCL20, performed as previously described, ${ }^{12}$ were in the median range of a larger cohort of MM patients without NF1 (data not shown). Accordingly, at the bone biopsy any significant morphological alterations were not observed in osteoclasts (Figure 1C).

Finally, the expression of p-ERK was analyzed by immunohistochemistry on bone biopsy to evaluate the possible activation of MAPK pathway. An intense p-ERK expression was demonstrated on MM cells (Figure 1D) with a positive staining of both endothelial cells and osteoblasts.

\section{Case Report \#2}

A 76-year-old man was referred to our center in November 2016 for a monoclonal gammopathy reported from 1992. His medical and surgical history included multiple cutaneous nodules, also presented in his father and son, cataract in left eye, bladder leiomyosarcoma treated with cystectomy and ileal conduit urinary diversion on 1999, myocardial infarction on 2005, vertebral and femoral osteoporosis diagnosed in 2014. He was asymptomatic, his blood count and renal function were normal and the serum IgG-kappa monoclonal protein was $1.7 \mathrm{gr} / \mathrm{dL}$. The general examination revealed multiple cutaneous sessile nodules with features of neurofibromas on the chest and at the midline of the back and more than six café-au-lait macules. The skeletal survey showed malacic vertebral collapse on D9 and D12, scoliosis with dystrophic and little curvature deformity, and right femur with congenital destroying lesion (Figure 2); no lithic lesions were observed. Lisch nodules were present in both eyes. The bone marrow biopsy contained 12\% PCs (Figure 2C). He was diagnosed with smoldering MM and NF1. In accordance to the guide- lines the patient was not treated and a follow up program was defined. To confirm the diagnosis of NF1 in both MM patients, a molecular testing was performed.

We found that patient 1 was heterozygous for the c.6855C $>\mathrm{A}$ (Tyr2285Ter) mutation, while patient 2 was heterozygous for the c.7838dupC (Lys2614GlufsTer20) mutation, both in NF1 gene. The two mutations were diagnosed both in genomic DNA from peripheral blood and from PCs. No further mutations were found (Figure 3).
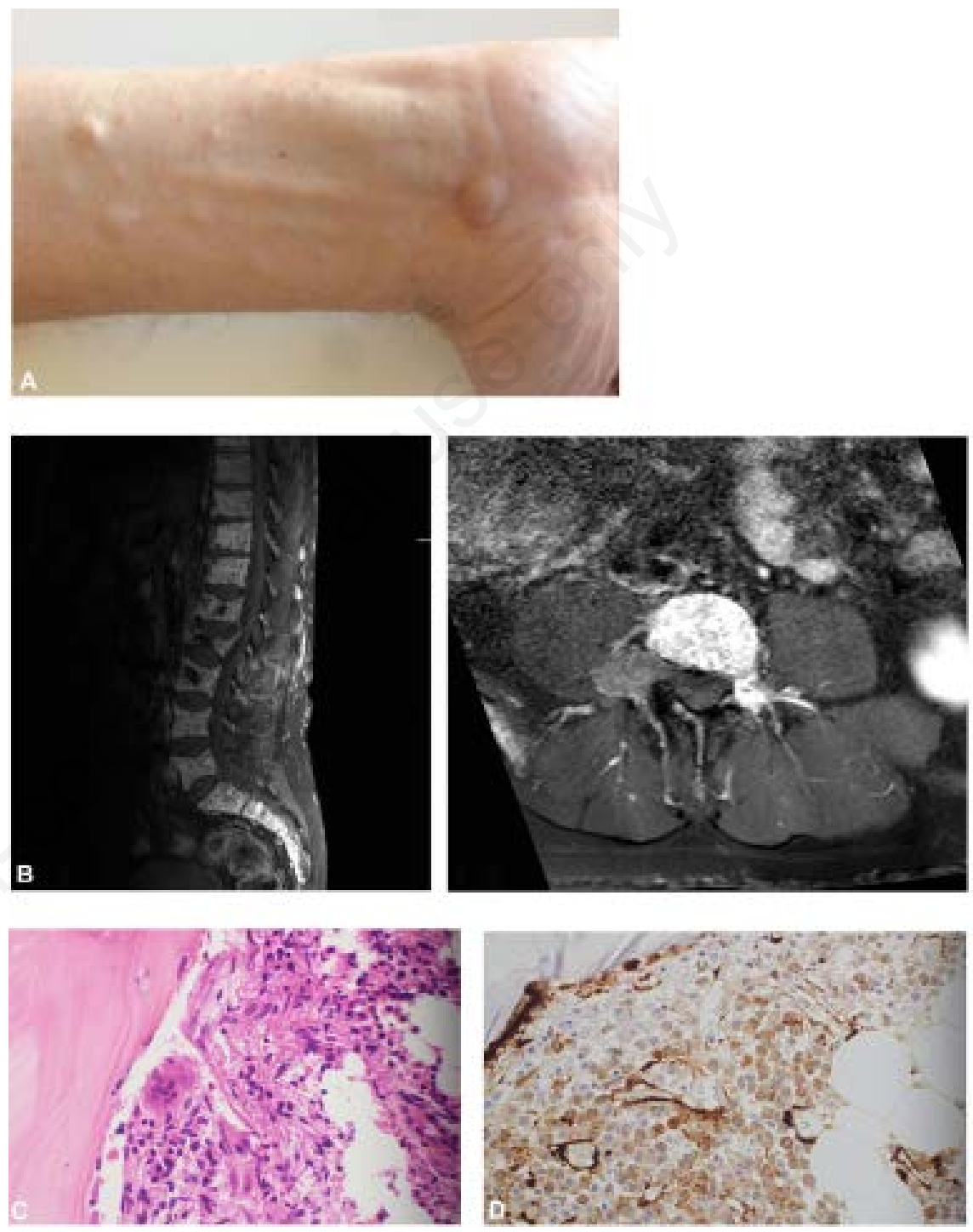

Figure 1. Clinical-radiological and histological features of case 1 with NF1 neurofibromatosis and multiple myeloma. Presence of several palpable nodules at the back of the hand and forearm (A). MRI of the spine. Sagittal view contrast enhanced T1-weighted image demonstrates a vertebral collapse on L2 (B, on the left). MRI axial view shows a heterogeneously enhancing dumbbell shaped neurofibroma at $\mathrm{L} 3$ level (B, on the right). Hematoxylin-eosin staining of a bone biopsy section evaluated by Optic Microscope (Olimpus BX51); Original magnification: 40X (C). p-ERK expression was analyzed by immunohistochemistry on bone biopsy. Rabbit monoclonal antibodies to p-ERK (Thr202/Tyr204, D13.14.4E, Cell Signaling) at 1:400 dilution were used. Immunostaining was done with polymeric system (Ultraview Universal DAB Setection Kit-Roche) according to the manufacture's specifications. Original magnification $40 \mathrm{X}$ (D). 
sis, were present only in the second case. Both patients did not show osteolytic lesions suggesting that the presence of NF1 phenotype apparently did not influence the development of MM bone disease despite the fact that osteoclast alterations have been previously reported in NF1 patient. $^{5}$

Bisphosphonates have been shown to increase bone density and reduce bone turnover in NF1 patients. ${ }^{13}$ Consistent with this, the patient treated with zoledronic acid has not developed bone fractures until now.

Genomic instability represents a main aspect of MM biology. Recently, wholegenome sequencing (WGS) and wholeexome sequencing (WES) studies revealed a more complex genetic dynamic model characterized by the presence, in the same patient, of distinct subclones at diagnosis which evolve over time through a linear or branching pathways. ${ }^{14-16}$ In this setting the most frequently activating mutations described involve RAS/MAPK and nuclear factor (NF)-kB pathways. MAPK pathway, including N-RAS, K-RAS, B-RAF, is affected by mutational events in elevated fraction of newly diagnosed MM patients ranging from $43 \%$ to $60 \%$ depending on the methods used for mutation detection. ${ }^{17}$ Notably the mutations of these driver genes were identified at both clonal and sub-clon- al levels demonstrating how these variations on the genetic architecture could represent an early event in the oncogenesis process or acquired late during disease progression. One study described the mutations of NFI gene, which is involved in the negative regulation of RAS signaling, in 2 of 67 MM patients. ${ }^{15}$ Interestingly one mutation was missense, while the other an indel mutation associated with the deletion of wild type allele.
As reported recently BRAF/RAS mutation status alone is not necessarily associated with MEK/ERK pathway activation and phosphorylated ERK1/2 has to be confirmed by immunohistochemistry to demonstrate a true activation of the prooncogenic signal. ${ }^{18}$ Noteworthy, in one patient, partially refractory to bortezomibbased induction therapy, a strong p-ERK expression was found, confirming a hyperactivation of RAS signaling. N-RAS muta-
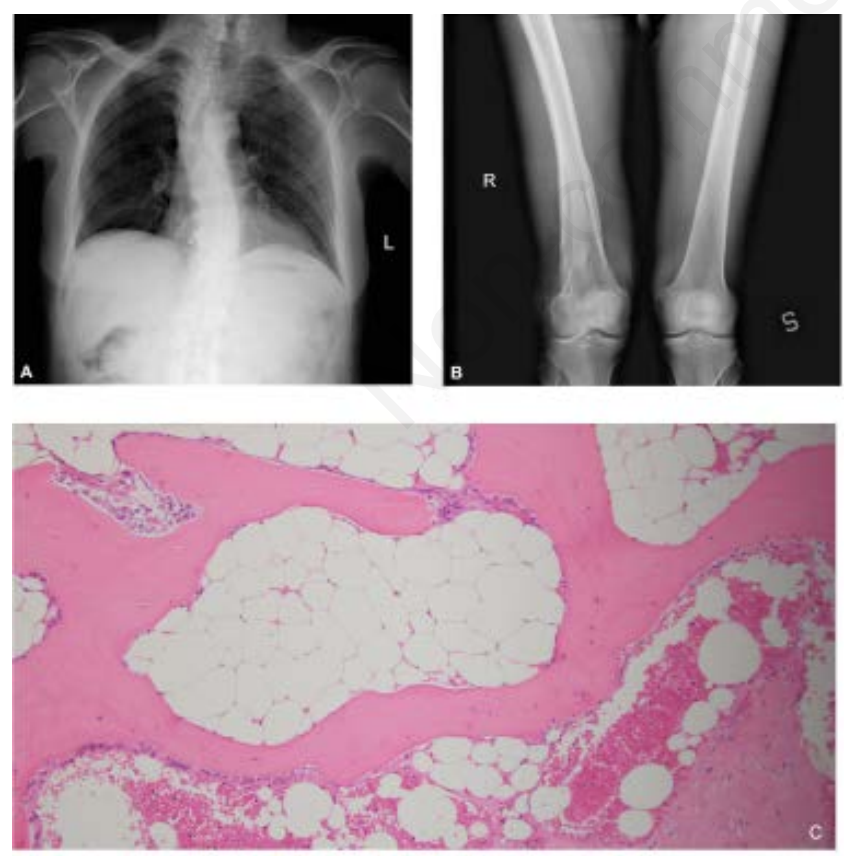

Figure 2. Radiological and histological feature of the case 2 with NF1 neurofibromatosis and smoldering myeloma. $X$ - ray images showed scoliosis of the spine (A-P view) with distrofic and little curvature deformity (A) and NF1 destroying lesion (A-P proximal view) in right femur (B). Hematoxylin-eosin staining of a bone biopsy section evaluated by Optic Microscope (Olimpus BX51); Original magnification $10 \mathrm{X}(\mathrm{C})$.

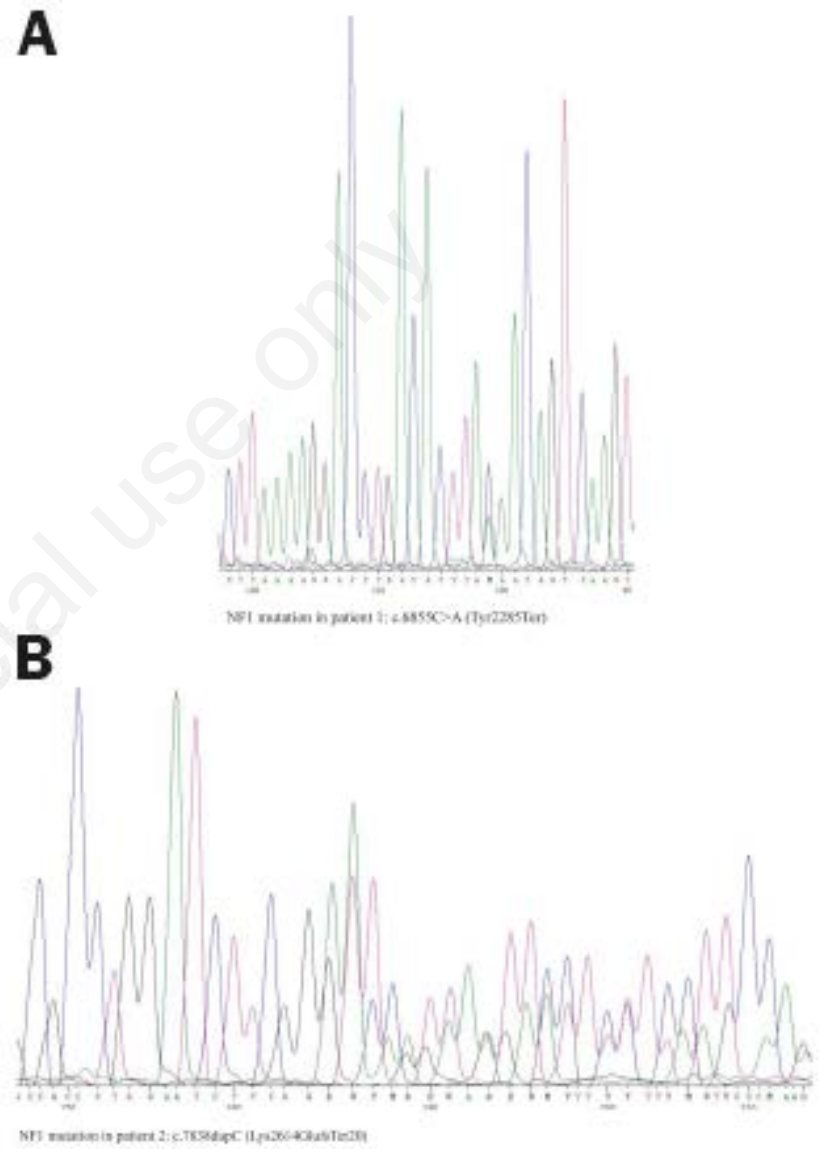

Figure 3. Mutations in NF-1 gene in the two cases of MM. DNA was extracted from whole blood and purified $\mathrm{CD}_{138}{ }^{+} \mathrm{PCs}$ with QIAamp DNA Blood Mini kit (Qiagen, Valencia, CA, USA) and purity was assessed by NanoDrop 2000 (Thermo Fisher Scientific, Waltham, MA, USA). DNA yields were determined using a double stranded DNA (dsDNA) broad range (BR) kit on a Qubit fluorometer (Thermo Fisher Scientific). Illumina TruSeq Custom Amplicon Kit was used to capture all exons, intron-exon boundaries, 5'- and 3'-UTR sequences and 50-bp flanking sequences of NF1 gene (RefSeq database, hg19 assembly, NM_001042492.2, NP_001035957.1). Manufacturer's instructions were used for the targeted capture library construction. Bioinformatic analysis was performed with VariantStudio software (Illumina). All the NF1 exons were obtained with at least 100 reads each. Genomic mutations were confirmed by Sanger sequencing in a CEQ2000XL (Beckman Coulter). Primers were designed using NCBI's Primer BLAST tool and the sequences were analyzed with the SeqMan software (DNAStar). Patient 1 (A) was heterozygous for the c.6855C $>\mathrm{A}$ (Tyr2285Ter) mutation, while patient 2 (B) was heterozygous for the c.7838dupC (Lys2614GlufsTer20) mutation, both in NF1 gene. 
tions have been associated to a lower response to bortezomib and worse prognosis in relapsed patients. ${ }^{19}$

In this report we speculate that NF1 haploinsufficiency could enhance RAS/ MAPK pathway signaling, inducing terminal ERK phosphorylation and contributing to the observed clinical behavior. Although the association of NF1 and MM is rarely described in literature a recent epidemiologic study on cancer incidence in 8003 NF1 patients reported a relative risk of $\mathrm{MM}$ of 2.4. ${ }^{20}$ On the other hand our data did not support a direct link between NF1 and MM pathogenesis. In conclusion these two cases of NF1 and MM suggest that in the work-up of NF1 patients, focused on the prevention of potential complications such as malignant evolution of benign tumors, a concomitant monoclonal gammopathy of undetermined significance should be carefully evaluated in order to recognize patient's risk of progression toward a symptomatic disease and establish an optimal frequency of follow up.

\section{References}

1. Hirbe AC, Gutmann DH. Neurofibromatosis type 1: a multidisciplinary approach to care. Lancet Neurol 2014; 13:834-43.

2. Ratner N, Miller SJ. A RASopathy gene commonly mutated in cancer: the neurofibromatosis type 1 tumour suppres sor. Nat Rev Cancer 2015;15:290-301.

3. Crawford AH, Schorry EK. Neurofibromatosis update. J Pediatr Orthop 2006;26:413-23.

4. Stevenson DA, Little D, Armstrong L, et al. Approaches to treating NF1 tibial pseudarthrosis: consensus from the Children's Tumor Foundation NF1 Bone Abnormalities Consortium. J Pediatr Orthop 2013;33:269-75.

5. Heervä E, Alanne MH, Peltonen S, et al. Osteoclasts in neurofibromatosis type 1 display enhanced resorption capacity, aberrant morphology, and resistance to serum deprivation. Bone 2010;47:58390.

6. Seitz S, Schnabel C, Busse B, et al. High bone turnover and accumulation of osteoid in patients with neurofibromatosis 1. Osteop Int 2010;21:119-27.

7. Lin AL, Gutmann DH. Advances in the treatment of neurofibromatosis-associated tumours. Nat Rev Clin Oncol 2013; 10:616-24.

8. Sartor C, Papayannidis C, Abbenante $\mathrm{MC}$, et al. A case report of acute myeloid leukemia and neurofibromatosis 1 . Hematol Rep 2013;5:28.

9. Gregory R, Hill J. von Recklinghausen neurofibromatosis and myelomatosis. Br J Clin Pract 1990;44:725-6.

10. Danilatou V, Liapi D, Psyllaki M, et al Neurofibromatosis type I and smoldering multiple myeloma: A case report. Hematology 2006;11:45-8.

11. Zengin N, Gönül $M$, Gül Ü, et al. Multiple myeloma in a patient with neurofibromatosis. Am J Hematol 2007; 82:772.

12. Palma BD, Guasco D, Pedrazzoni M, et al. Osteolytic lesions, cytogenetic features and bone marrow levels of cytokines and chemokines in multiple myeloma patients: Role of chemokine (C-C motif) ligand 20. Leukemia 2016;30: 409-16.

13. Heerva E, Huilaja L, Leinonen P, et al.
Follow-up of six patients with neurofibromatosis 1-related osteoporosis treated with alendronate for 23 months. Calcif Tissue Int 2014;94:608-12.

14. Lohr JG, Stojanov P, Carter SL, et al. Widespread genetic heterogeneity in multiple myeloma: implications for targeted therapy. Cancer Cell 2014;25:91101.

15. Bolli N, Avet-Loiseau H, Wedge DC, et al. Heterogeneity of genomic evolution and mutational profiles in multiple myeloma. Nature Commun 2014;5

16. Walker BA, Boyle EM, Wardell CP, et al. Mutational spectrum, copy number changes, and outcome: results of a sequencing study of patients with newly diagnosed myeloma. J Clin Oncol 2015; 2059:503.

17. Lionetti M, Barbieri M, Todoerti K, et al. Molecular spectrum of BRAF, NRAS and KRAS gene mutations in plasma cell dyscrasias: implication for MEK-ERK pathway activation. Oncotarget 2015;6:24205-17.

18. Xu J, Pfarr N, Endris V, et al. Molecular signaling in multiple myeloma: association of RAS/RAF mutations and MEK/ERK pathway activation. Oncogenesis 2017;6:e337.

19. Mulligan G, Lichter DI, Di Bacco A, et al. Mutation of NRAS but not KRAS significantly reduces myeloma sensitivity to single-agent bortezomib therapy. Blood 2014;123:632-9.

20. Seminog OO, Goldacre MJ. Risk of benign tumours of nervous system, and of malignant neoplasms, in people with neurofibromatosis: population-based record-linkage study. Br J Cancer 2013; 108:193-8. 